Available online at:

https://acta-acustica.edpsciences.org

\title{
Directivity pattern of the sound radiated from square stepped-plate radiators
}

\author{
$\mathrm{He} \mathrm{Xiping}^{*}$ and Li Na \\ Key Laboratory of Ultrasonics, Shaanxi Normal University, Xian, Shaanxi 710062, PR China
}

Received 30 March 2020, Accepted 14 July 2020

\begin{abstract}
A square flat-plate radiator driven by a longitudinal vibrator at the resonant frequency of its fourth-order natural vibration mode at its center for the generation of highly directional ultrasonic radiation in air is studied herein. The radiator was able to create a bending vibration mode whose four straight nodal lines were located at a $45^{\circ}$ angle on each side. To obtain highly directional sound, the surrounding area of the four right isosceles triangles formed by a nodal line and two sides is modified to be raised one by half the wavelength of sound radiated in the propagation medium so that surface elements vibrate in phase. Then, the relation among the frequencies, nodal lines of the rhombus mode, and geometrical dimensions of the radiator is determined. The calculated directivity pattern of the stepped-plate radiator is equivalent to that of its corresponding theoretical piston, i.e., a stepped plate is a piston-like radiator. Results show that the measured vibrational-mode shape of the stepped-plate radiator is consistent with the calculated one, and the calculated directivity pattern is almost similar to the measured one.
\end{abstract}

Keywords: Square stepped-plate radiator (SSPR), Flexural vibration, Response mode, Directivity pattern

\section{Introduction}

High-power ultrasonic transducers, commonly used for gases, are aerodynamic systems of various types such as whistles and sirens [1-6], in which the acoustic energy is provided by a gas jet. However, the sounds they emit are complex, the efficiencies are low, and the directivities are poor in these devices. In practical applications such as detection of objects and measurement of distances the impedance mismatch in air coupling transducers between the transducer surface and air is large, resulting in only a small amount of energy transmission. Most of the energy loss occurs during the transformation of energy from the transducer to air.

To obtain highly directional sound and good impedance matching to air or fluids and multiphase media, GallegoJuárez et al. introduced a square stepped-plate, whose raised step height at coincident nodal lines with the edges is half the wavelength of the radiated sound. They also developed several other types of airborne sonic and ultrasonic power generators [7-10], which comprise a flexural vibrating circular-plate radiator, or a rectangular plate radiator with a stepped profile, driven at its center by a piezoelectric vibrator. These are required for many applications such as for detecting or locating distant objects [11], dehydration of food for preservation [12, 13], ultrasonic

*Corresponding author: hexiping@snnu.edu.cn precipitation, ultrasonic levitation, ultrasonic cohesion, ultrasonic debubbling, and other ultrasonic processing needs [14-20]. To suppress the bending motion of the radiation plate and set the transducer close together, Yamamori et al. changed the radiating surface of a bolt-clamped Langevin-type transducer used in ultrasonic cleaning from round to square [21].

A flexural vibration square-plate radiator with steps having in-phase vibration driven at its center by a longitudinal vibration transducer is thoroughly studied herein. A square flat plate driven by a longitudinal vibration transducer with the same resonant frequency at its center was able to create a flexural vibration mode whose four nodal lines were located at a $45^{\circ}$ angle on each side (no such a mode in the natural modes of the square flat plate). To obtain highly directional sound, the surrounding area of the four isosceles triangles formed by a nodal line and two sides was modified to be raised as a Square Stepped-Plate Radiator (SSPR) whose thickness increment was approximately half the wavelength of the radiated sound in the propagation medium, enabling surface elements to vibrate in phase and avoiding phase cancellation (the profile of the SSPR is shown in Fig. 4a).

The calculated radiation pattern of the stepped radiator was better than that of the flat one, whose configuration was similar to that of the theoretical piston. The measured vibrational-mode shape of the stepped-plate radiator was consistent with the calculated one. This type of SSPR 
oscillating in simpler bending mode is expected to be used in ultrasound distance and level measurements in stock owing to its extensive surface exhibiting good impedance matching to air, its high directivity, and its high strength and amplitude at its operating frequency when driven by a longitudinal sandwiched vibrator.

\section{Theoretical calculations}

A square thin plate with a free edge is shown in Figure 1. Waller tested the natural vibration modes of a square plate $[22,23]$ and found that the natural modes of a square plate are complex and irregular. Figure 2 shows 1st, 2nd, 3rd and 4th mode shapes of a Square Flat-Plate Radiator (SFPR).

\subsection{Response vibration mode}

The side length $l$ of the SFPR was $50 \mathrm{~mm}$ and its thickness $h$ (it is also called thickness of the base plate) was $6 \mathrm{~mm}$. The material used was steel with a Poisson ratio of $v=0.28$, Young's modulus of $E=1.96 \times 10^{11} \mathrm{~N} / \mathrm{m}^{2}$, and a density of $\rho=7.91 \times 10^{3} \mathrm{~kg} / \mathrm{m}^{3}$. The corresponding frequency $f$ of the profile of its fourth vibration mode, as shown in Figure 2d, was 19,332 Hz.

Connection of the SFPR center by a bolt to a longitudinal vibration transducer with the same resonant frequency $f=19,332 \mathrm{~Hz}$ formed a compound vibration system. The commercial finite element program ANSYS was employed to perform the computations. The 10-node quadratic element solid187 was used to model the radiators. The flat radiator was divided into 449 elements. Through a harmonic response analysis, the vibration mode shape of the radiator with four straight nodal lines intersecting two adjacent sides can be obtained at frequency $f_{\mathrm{e} 1}=19,006 \mathrm{~Hz}$, as shown in Figure 3a.

The obtained mode is called the rhombus response mode that does not exist in the eigenmodes of the SFPR, i.e., it can be created only if driven by a longitudinal vibrator. The coordinates of the eight nodes can be calculated from the vibration displacement perpendicular to the square-plate plane along the four sides. The nodes are shown in Figure $3 \mathrm{~b}$. The coordinates of node numbers 1-8 in units of meters were $(0.025,0.004,0),(0.004,0.025,0),(-0.004,0.025,0),(-0.025$, $0.004,0), \quad(-0.025,-0.004,0), \quad(-0.004,-0.025,0), \quad(0.004$, $-0.025,0)$, and $(0.025,-0.004,0)$, respectively. The connections between nodes 1 and 2, 3 and 4, 5 and 6 , and 7 and 8 were just the four nodal lines, and each line intersected two sides of the plate with an angle of $45^{\circ}$, being an isosceles right triangle.

The surface elements on two sides of a straight nodal line vibrated out of phase, leading to cancellation. The SFPR (\#I) can be improved as a stepped radiator (\#II). The raised step was added at the section surrounded by the four isosceles triangles formed by one straight nodal line and two adjacent sides, whose height was equal to the half the wavelength of the sound radiated in the propagation medium. The original straight nodal lines will be expanded outside for the raised square stepped plate driven by the

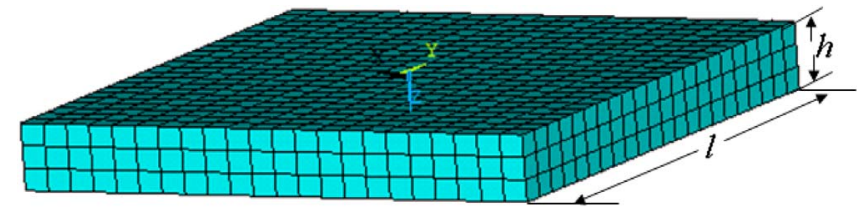

Figure 1. The finite element model of a square thin plate.

transducer at the primary frequency. Adjusting the position and height of the step can compensate for the flexural vibration and ensure that the edges of the step coincide with the location of the nodal lines. After adjustment, the coordinates of nodes $1-8$ were $(0.025,0.009,0),(0.009,0.025,0),(-0.009$, $0.025,0),(-0.025,0.009,0),(-0.025,-0.009,0),(-0.009$, $-0.025,0),(0.009,-0.025,0)$, and $(0.025,-0.009,0)$, respectively. Here, the height of the step was $h_{1}=c / 2 f_{\mathrm{e} 2}=$ $0.01 \mathrm{~m}$, where $c$ is the propagation velocity of sound waves in air $\left(\right.$ at $\left.15{ }^{\circ} \mathrm{C}\right)$, as shown in Figure 4a. Figure 4b shows the response vibration mode at $f_{\mathrm{e} 2}=15,751 \mathrm{~Hz}$.

\subsection{Directivity pattern}

Let us assume that the radiator is mounted flush with an infinite baffle, with the coordinate origin $O$ taken as the center of the upper surface as shown in Figure 5. The radiator driven by a longitudinal vibrator radiated waves to the front half of the baffle space. Observation point $Q\left(x_{0}, y_{0}, z_{0}\right)$ was located in the far field. The radiator was divided into multiple elements, each of which has an infinitesimal area of $\mathrm{d} s=\mathrm{d} x \mathrm{~d} y$. According to the Rayleigh integral formula, the radiation pressure at point $Q$ generated by the infinitesimal area $d s$ can be expressed as follows

$$
\mathrm{d} p=j \frac{k \rho_{0} c_{0}}{2 \pi R} u(x, y) \mathrm{e}^{j(\omega t-k R)} \mathrm{d} s,
$$

where $R=\sqrt{\left(x_{0}-x\right)^{2}+\left(y_{0}-y\right)^{2}+z_{0}^{2}}$ is the distance between the infinitesimal area $\mathrm{d} s$ and the observation point $Q$, and $u(x, y)$ is the vibration velocity amplitude of the infinitesimal area $\mathrm{d} s$.

The radiation pressure generated from the whole radiator can be expressed as follows

$$
p=j \frac{k \rho_{0} c_{0}}{2 \pi} \iint \frac{u(x, y)}{R} e^{j(\omega t-k R)} \mathrm{d} s
$$

$R$ in the denominator can be approximately replaced with $r$ (the distance between the coordinate origin and the observation point $Q$ ) for $r$ is much greater than the side length of the SFPR. From Figure 5, $R$ in the exponent (i.e., the phase section) can be approximately expressed by the following equation

$$
R=r-\frac{x_{0} x+y_{0} y}{r} .
$$

Direction cosines at point $Q$ are expressed as follows

$$
\cos \alpha=\frac{x_{0}}{r}, \cos \beta=\frac{y_{0}}{r}, \cos \gamma=\frac{z_{0}}{r},
$$




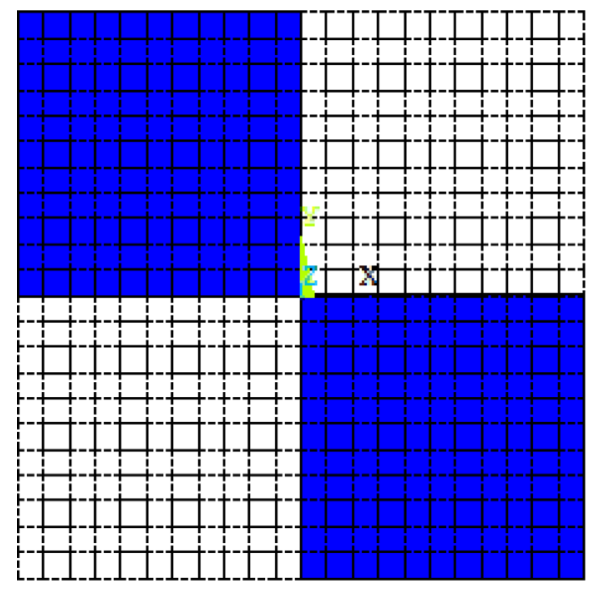

(a)

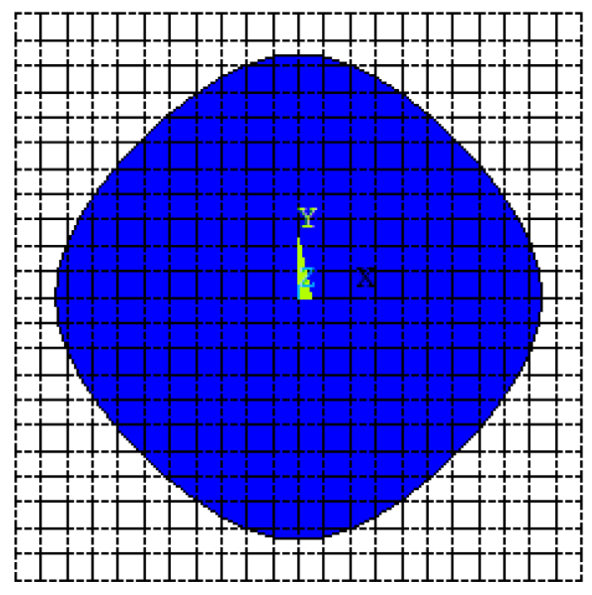

(c)

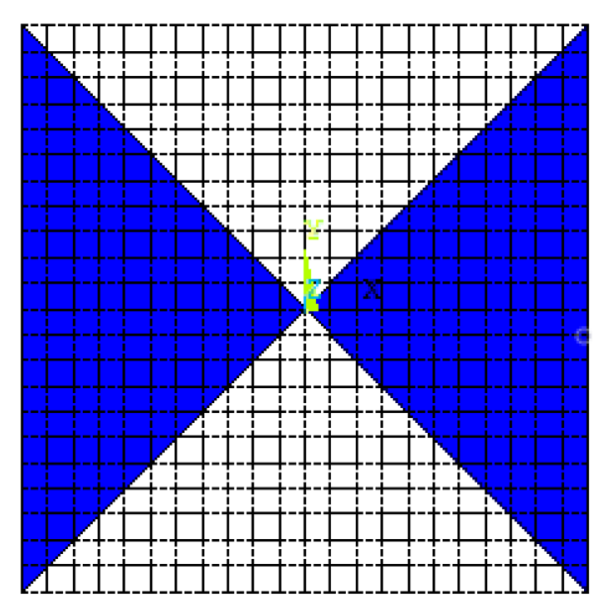

(b)

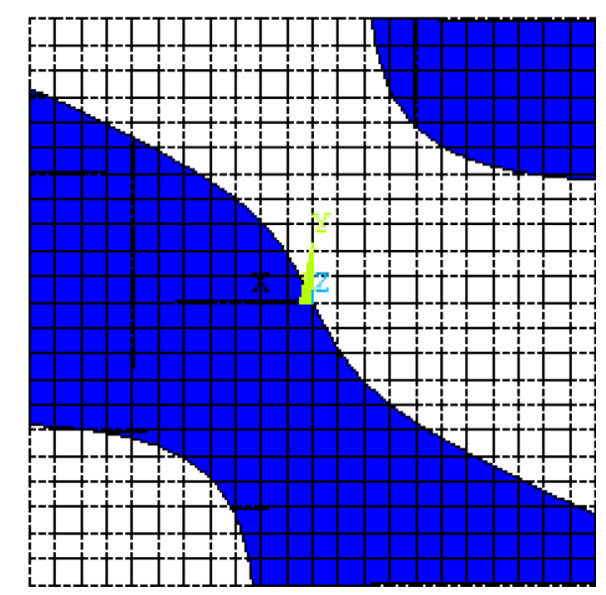

(d)

Figure 2. The first four natural vibration mode shapes of an SFPR (a) 1st, (b) 2nd, (c) 3rd, and (d) 4th.

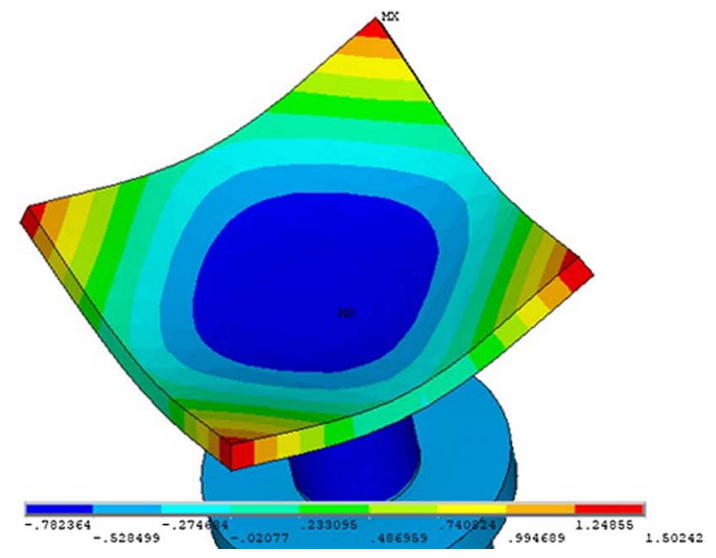

(a)

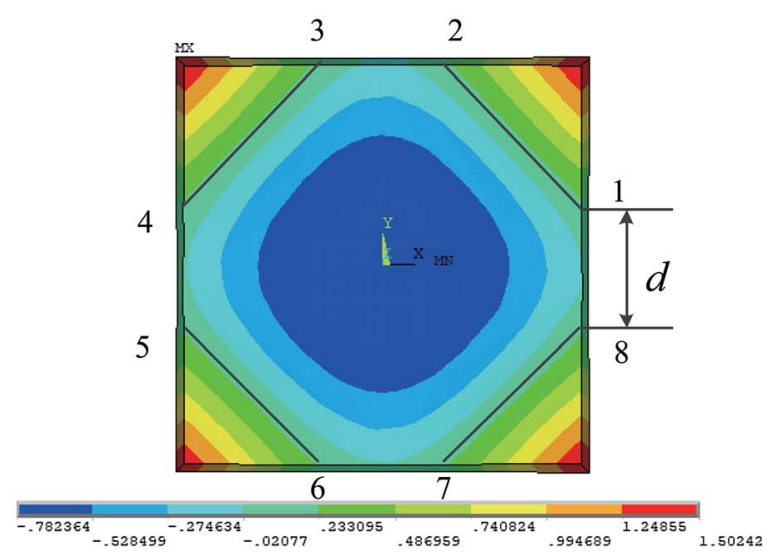

(b)

Figure 3. A response vibration mode obtained at $19,006 \mathrm{~Hz}$ by driving center of the SFPR with a longitudinal vibration transducer. (a) The response vibration mode, (b) position of the four straight nodal lines. 


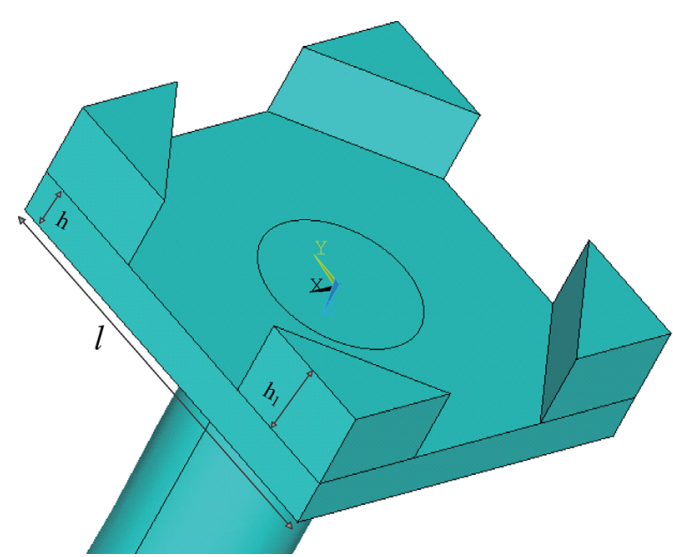

(a)

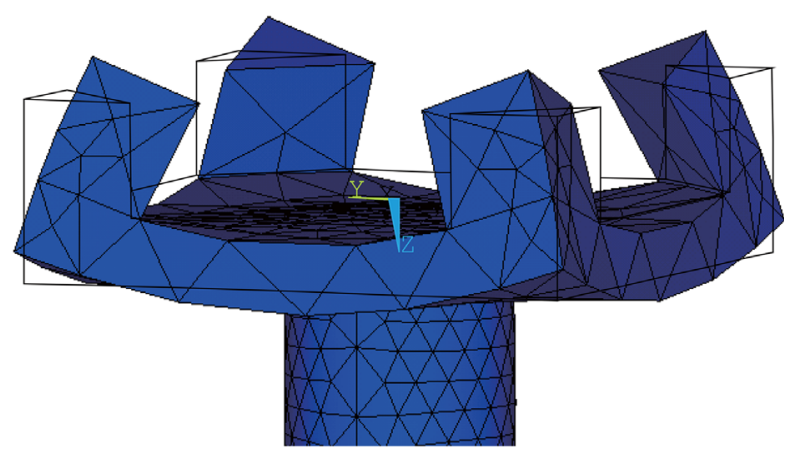

(b)

Figure 4. A square stepped plate and its response vibration mode. (a) The stepped plate, (b) the vibration mode at $f_{\mathrm{e} 2}=15,751 \mathrm{~Hz}$.



Figure 5. Calculation of the radiation pressure generated by the square-plate radiator.

and the relations between the direction cosines and the sphere coordinates are shown below

$$
\cos \alpha=\sin \theta \cos \varphi, \cos \beta=\sin \theta \sin \varphi .
$$

Substituting the above equations into equation (2) yields the following equation

$$
p=j \frac{k \rho_{0} c_{0}}{2 \pi r} \mathrm{e}^{j(\omega t-k r)}\left(b_{1}+j b_{2}\right),
$$

where

$$
\begin{aligned}
b_{1}= & \iint u(x, y)(\cos (k x \sin \theta \cos \phi) \cos (k y \sin \theta \sin \phi) \\
& -\sin (k x \sin \theta \cos \phi) \sin (k y \sin \theta \sin \phi)) \mathrm{d} x \mathrm{~d} y, \\
b_{2}= & \iint u(x, y)(\cos (k x \sin \theta \cos \phi) \sin (k y \sin \theta \sin \phi) \\
& +\cos (k y \sin \theta \sin \phi) \sin (k x \sin \theta \cos \phi)) \mathrm{d} x \mathrm{~d} y .
\end{aligned}
$$

Discretization of $b_{1}$ and $b_{2}$ provides the following equations

$$
\begin{aligned}
B_{1}= & \sum_{i=1}^{N} u_{i}(x, y)\left(\cos \left(k x_{i} \sin \theta \cos \phi\right) \cos \left(k y_{i} \sin \theta \sin \phi\right)\right. \\
& \left.-\sin \left(k x_{i} \sin \theta \cos \phi\right) \sin \left(k y_{i} \sin \theta \sin \phi\right)\right) \Delta s_{i},
\end{aligned}
$$

$$
\begin{aligned}
B_{2}= & \sum_{i=1}^{N} u_{i}(x, y)\left(\cos \left(k x_{i} \sin \theta \cos \phi\right) \sin \left(k y_{i} \sin \theta \sin \phi\right)\right. \\
& \left.+\cos \left(k y_{i} \sin \theta \sin \phi\right) \sin \left(k x_{i} \sin \theta \cos \phi\right)\right) \Delta s_{i},
\end{aligned}
$$

where $u_{i}$ represents the vibration velocity of segment number $i$, and $x_{i}$ and $y_{i}$ represent the coordinates of segment number $i$. Therefore, the amplitude of radiation pressure is as follows

$$
|p|=\frac{k \rho_{0} c_{0}}{2 \pi r} \sqrt{B_{1}^{2}+B_{2}^{2}} .
$$

According to the definition,

$$
D(\theta, \phi)=\frac{|P|_{\theta}}{|P|_{\theta=0}} .
$$

By combining with equation (4), the directivity formula can be expressed as follows

$$
D(\theta, \phi)=\left|\frac{\sqrt{B_{1}^{2}+B_{2}{ }^{2}}}{\omega \sum_{i=1}^{N} q_{i} \Delta s_{i}}\right| .
$$

The displacement $\xi$ and vibration velocity $v$ of the plate are related as follows

$$
v=\frac{\partial \xi}{\partial t}=\frac{\partial\left(q e^{j \omega t}\right)}{\partial t}=j \omega q e^{j \omega t}=u e^{j \omega t}, \quad \text { so } \quad u=j \omega q .
$$

The commercial finite element program ANSYS was employed to perform the computations. The vibration displacements $q_{i}$ of each infinitesimal can be obtained by the finite element modal calculation. Then, extraction of the displacements of each infinitesimal at the plate plane and their substitution into equation (6) allowed the radiation directivity patterns of the two radiators to be obtained through the programming calculation, as shown in Figure 9 in Section 3.2. There is one issue that needs to be illustrated. The radiation surfaces were not on the same flat plane (the radiation surfaces on the steps were located at 
a half wavelength above the flat plane) and the surface elements still remained vibrating in the counter phase. However, the radiation of the steps and the flat plane were in phase. Therefore, considering the symbol of the displacement on the flat plate as the opposite sign in counter-phase zones (actually these zones were modified as steps) or considering all displacement values on the flat plate as absolute ones. Displacement of the stepped-plate radiator can be calculated using the displacement of the flat plate.

\section{Experimental measurement of the response mode and directivity patterns}

\subsection{Response mode}

The flat and the stepped-plate steel radiators I and II described above were fabricated (Figure 6). The II\# SSPR can be obtained by milling a thick square plate.

Radiators I and II were excited with sinusoidal signals provided by the Polytec PSV-400 Scanning Vibrometer. The laser sensor of the Polytec PSV-400 Scanning Vibrometeris scanned on the surface of the radiators (the stepped surface faced the longitudinal vibrator and its flat back surface faced the laser sensor to be scanned for radiator II), and the velocity spectra and the modes corresponding to the peak values can be obtained, as shown in Figures $7 \mathrm{a}$ and $7 \mathrm{~b}$. Accordingly, the frequencies corresponding to the modes for radiators I and II were $f_{\mathrm{t} 1}=18,563 \mathrm{~Hz}$ and $f_{\mathrm{t} 2}=15,547 \mathrm{~Hz}$, respectively.

\subsection{Directivity patterns}

The schematic of the experimental setup used to perform the directivity measurements is shown in Figure 8. The plate of the transducer was placed in a large flat foamed plastic baffle. The acoustic pressure probe (a type uc-29 microphone $\frac{1}{4}$ in. diameter, its working frequency ranged from $20 \mathrm{~Hz}$ to $100 \mathrm{kHz}$ ) was placed in the far field in front of the plate. The frequency range of a type NA- 42 sound level measuring amplifier was from $1 \mathrm{~Hz}$ to $100 \mathrm{kHz}$. Both type uc-29 microphone and type NA-42 sound level measuring amplifier are manufactured by Rion Co., Ltd., Japan. Measurements were obtained in an unobstructed environment, which was on the roof of our laboratory building. Each test was performed across $90^{\circ}$ for the transducer and mirrored to complete the plot, as shown in Figure 9. The dotted and solid lines represent the directivity patterns of the square flat and stepped radiators I and II, respectively.

\section{Analysis and discussion}

\subsection{Calculation and measurement}

The calculated frequency of the fourth-order natural vibration of the SFPR I was $f=19,332 \mathrm{~Hz}$, and the response mode, as shown in Figure 3b, did not exist in its eigenmodes. It could be only obtained while exciting the SFPR with the same longitudinal vibration frequency, which corresponded to the calculated frequency of $f_{\mathrm{e} 1}=19,006 \mathrm{~Hz}$. The tested

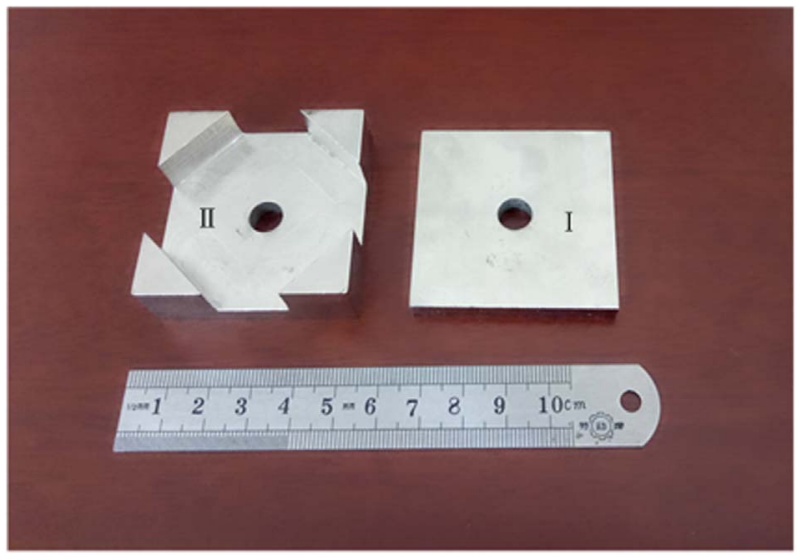

Figure 6. Fabricated square-plate radiators I and II.

response mode of the SFPR at $f_{\mathrm{t} 1}=18,563 \mathrm{~Hz}$ is shown in Figure $7 \mathrm{a}$. It can be seen from Figures $3 \mathrm{~b}$ to $7 \mathrm{a}$ that the calculated response mode was similar to the tested one, the frequency error being $\Delta_{1}=\left|f_{e 1}-\mathrm{f}_{t 1}\right| / f_{t 1}=2.3 \%$. The stepped-radiator II was modified from the flat-plate radiator I. For radiator II, the nodal lines were extended while adding the step to the surface of the original radiator I. However, after adjustment, the nodal line positions were consistent with those of the measured step edges, and the frequency error was $\Delta_{2}=\left|f_{\mathrm{e} 2}-f_{t 2}\right| / f_{t 2}=1.3 \%$.

The dashed-dot, dashed, and solid lines shown in Figure 9a show the calculated directivity patterns of the flat, theoretical piston, and square stepped radiators, respectively. Here, a theoretical piston means all portions of the SFPR vibrated in phase with the same amplitude, whose side length was the same as that of the stepped plate. It can be seen from Figure 9a for the SSPR II that there exist a sharper major lobe and some side lobes. However, the side lobes are minor compared to the main lobe, demonstrating that the sound energy distribution was concentrated. The SSPR II exhibited good directionality. As expected, the calculated directivity pattern of a steppedplate radiator is equivalent to that of its corresponding theoretical piston, i.e., a stepped plate is a piston-like radiator. However, for the SFPR I, there is no major lobe along its acoustic axis direction, demonstrating that the SFPR has poor directionality.

Comparisons of Figures 9a and 9b show that the calculated directivity patterns are almost similar to those of the measured ones. However, there are certain differences between the measured directivity patterns and the calculated ones. For example, the major lobes of the former are wider than those of the latter. The first reason for this difference is that the actual area of the flat foamed plastic baffle mounted in the experiment was finite rather than infinite, i.e., radiation from the back of the radiators may affect the acoustic field in front of the radiators. The second reason is that the diffraction field, which was generated by the unbaffled upper surface at the outer edge by the pressure from the surface underneath, may affect the acoustic field [24]. The third reason for this difference is that the standard values of the plate material parameters were different from 


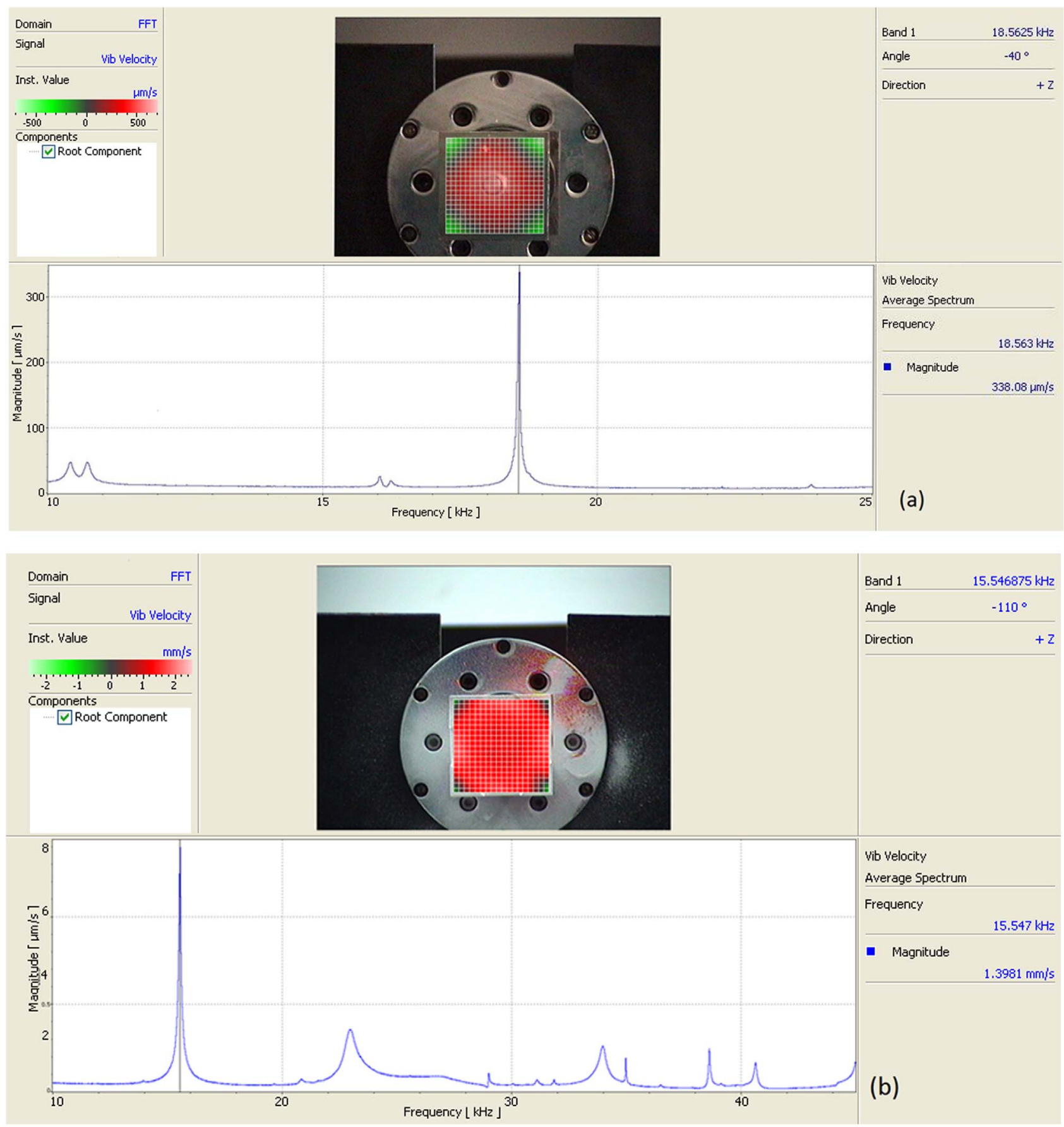

Figure 7. The vibration velocity spectra and the modes at the peak values of two radiators. (a) Radiator I, (b) Radiator II.

those of the practical values of the material. Finally, manufacturing errors and misalignment during the assembly process may also contribute to this discrepancy.

\subsection{Relation among the frequency, nodal lines of the rhombus mode, and geometrical dimensions of the SFPR}

The material used for the SFPR was steel. The thickness $h$ of the plate radiator was $6 \mathrm{~mm}$. To further illustrate the relation among the frequency, nodal lines of the rhombus mode, and geometrical dimensions of the radiator, theoretical calculations were conducted and the results are listed in Table 1. From the table, one can see that, with increasing side length of the radiator, the frequency of the fourth-order natural mode decreased when the thickness of the plate was held constant; the frequency $f_{\mathrm{e} 1}$ corresponding to the rhombus response mode decreased if driven at its center by a longitudinal vibrator at the frequency $f$ of the fourth-order natural mode, and the distance between the 


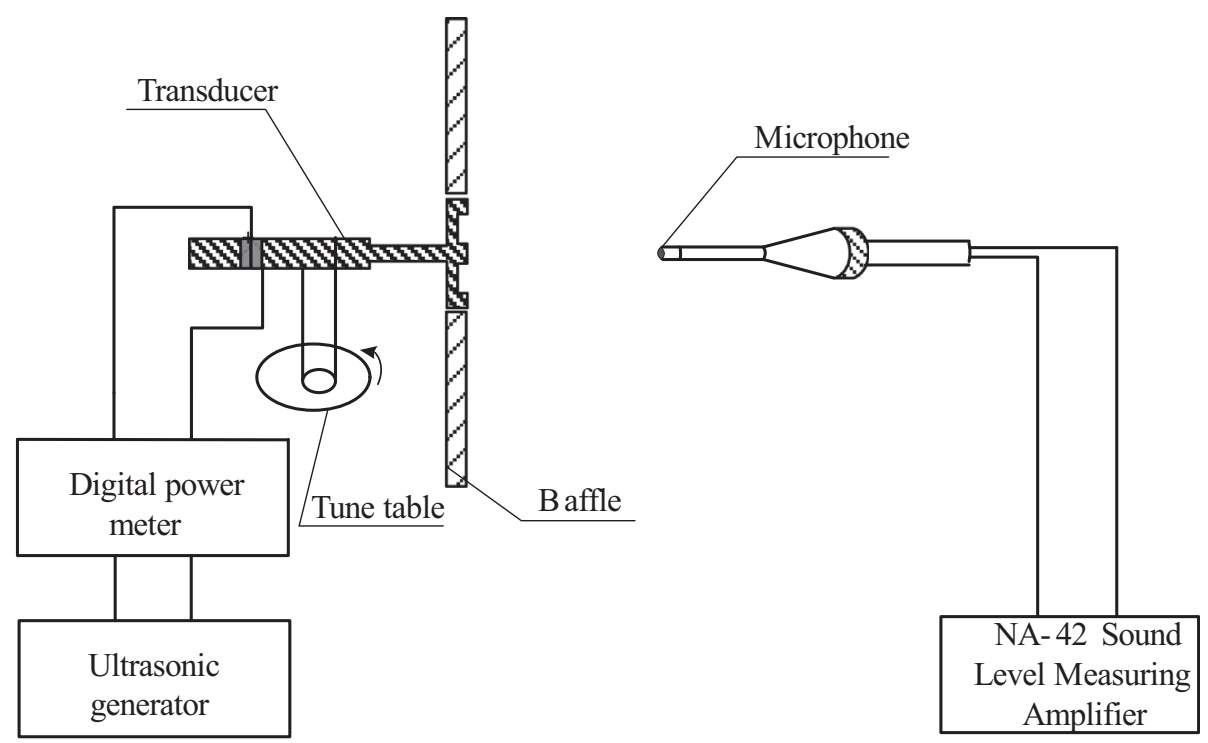

Figure 8. Experimental setup for the measurement of directivity pattern.

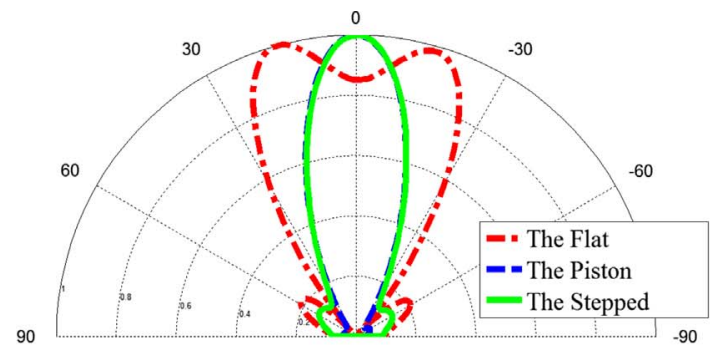

(a)

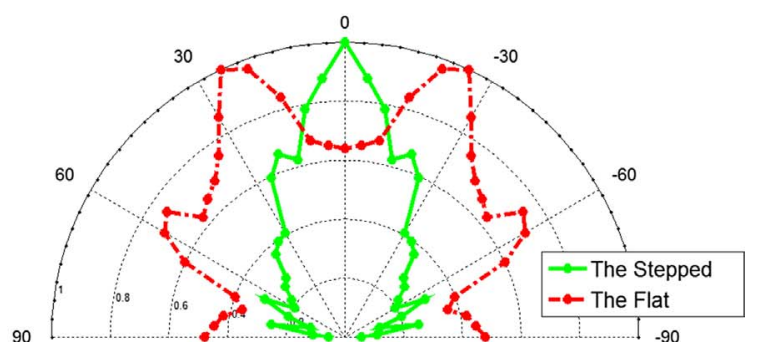

(b)

Figure 9. The directivity patterns of the square plate radiators. (a) The calculated, (b) the measured.

Table 1. Relation among the frequency, nodal lines of the rhombus response mode, and geometrical dimensions of the SFPR.

\begin{tabular}{lccccccccc}
\hline$l(\mathrm{~mm})$ & 60 & 65 & 70 & 75 & 80 & 85 & 90 & 95 \\
\hline$f(\mathrm{kHz})$ & 13.80 & 11.86 & 10.29 & 9.02 & 8.00 & 7.07 & 6.33 & 5.70 \\
$f_{\mathrm{e} 1}(\mathrm{kHz})$ & 12.97 & 11.11 & 9.60 & 8.41 & 7.39 & 6.51 & 5.86 & 5.28 & 4.76 \\
$d(\mathrm{~mm})$ & 10.08 & 10.60 & 10.92 & 11.70 & 12.12 & 12.60 & 13.30 & 13.68 & 14.40 \\
\hline
\end{tabular}

Table 2. Relation between the rhombus mode and the thickness of plate.

\begin{tabular}{lccccccccc}
\hline$h(\mathrm{~mm})$ & 6 & 6.5 & 7 & 7.5 & 8 & 8.5 & 9 & 9.5 \\
\hline$f(\mathrm{kHz})$ & 19.32 & 20.66 & 21.95 & 23.19 & 24.39 & 25.51 & 26.59 & 27.34 \\
$f_{\mathrm{e} 1}(\mathrm{kHz})$ & 18.36 & 19.44 & 20.62 & 22.22 & 22.80 & 23.65 & 24.54 & 25.32 & 26.51 \\
$d(\mathrm{~mm})$ & 4.10 & 3.80 & 3.50 & 2.50 & 3.30 & 3.10 & 3.00 & 2.00 & 1.50 \\
\hline
\end{tabular}

first and eighth nodes (as shown in Fig. 3b) increased, i.e., the nodal lines expanded outside.

By keeping the side length $(l=50 \mathrm{~mm})$ of the radiator constant, the frequency of the fourth-order natural mode increased with increasing thickness of the plate $h$, the frequency $f_{\mathrm{e} 1}$ corresponding to the rhombus response mode increased if driven at its center by a longitudinal vibrator at frequency $f$ of the fourth-order natural mode, and the distance $d$ between the first and eighth nodes (as shown in
Fig. 3b) decreased, i.e., the nodal lines shrank inside, as shown in Table 2.

\section{Conclusions}

In this paper, an SSPR was introduced to improve an SFPR, and the response vibration mode and the directivity pattern were calculated. The calculated and test results 
were basically consistent. To sum up the results obtained herein, the following conclusions can be drawn.

1. If the SFPR was driven at its center by a longitudinal vibrator with the same resonant frequency as its fourth-order natural one, a new rhombus response mode appeared that did not exist in the eigenmodes of the SFPR whose four straight nodal lines were located at a $45^{\circ}$ angle on each side.

2. The SSPR can serve as an improved form over the flat-plate radiator through calculation of the position of the straight nodal lines of the rhombus response mode. Its mechanical process was simple, and its beam pattern configuration of sound radiation was highly directional, which was equivalent to that of a theoretical piston with the same size of the square piston plate radiator.

3. The flexural resonant frequency of the SSPR could be applied to long-distance range and level measurement in stock. Similarly, the larger size of the square stepped radiator was also highly directional, and it can be used in various applications such as ultrasonic cleaning, ultrasonic defoaming, and ultrasonic drying.

\section{Conflict of interest}

Author declared no conflict of interests.

\section{Acknowledgments}

This work was supported by the National Natural Science Foundation of P. R. China (Grant No. 11774211).

\section{References}

1. B. Vonnegut: A Vortex whistle. Journal of the Acoustical Society of America 26 (1954) 18-20.

2. C. Robert: Chanaud: Experiments concerning the Vortex Whistle. Journal of the Acoustical Society of America 35 (1963) 953-960.

3. I. Michelson: Theory of Vortex Whistle. Journal of the Acoustical Society of America 27 (1955) 930-931.

4. S. Narayanan, P. Bhave, K. Srinivasan, K. Ramamurthi, T. Sundararajan: Spectra and directivity of a Hartmann whistle. Journal of Sound and Vibration 321 (2009) 875-892.

5. P. Greguss: The applications of air-borne and liquid-borne sounds to industrial technology. Ultrasonics 2 (1964) 5-10.

6. G. John: Powell: Siren. Journal of the Acoustical Society of America 83 (1988) 399.

7.A. Barone, J.A. Gallego: Flexural vibrating free-edge plates with stepped thickness for generating high directional ultrasonic radiation. Journal of the Acoustical Society of America 51 (1972) 953-959.

8. J.L. San Emeterio, J.A. Gallego-Juárez, G. Rodriguez-Corral: High axisymmetric modes of vibration of stepped circular plates. Journal of Sound and Vibration 114 (1987) 495-505.

9. J.A. Gallego-Juárez, G. Rodriguez, V. Acosta, E. Riera: Power ultrasonic transducers with extensive radiators for industrial processing. Ultrasonics Sonochemistry 17 (2010) 953-964.

10. J.A. Gallego-Juárez, G. Rodriguez, S.E. Riera, F.V. Martinez, C.C. Pozuelo, V.M. Acosta: Recent developments in vibrating-plate macrosonic transducers. Ultrasonics 40 (2002) 889893.

11. J.A. Gallego-Juárez, G. Rodriguez, J.L. San Emeterio, P.T. Sanz, J. C. Lázaro: An acoustic transducer system for longdistance ranging applications in air. Sensors and Actuators A: Physical 37-38 (1993) 397-402.

12. S.F. Blanco, S.E. Riera, V.M. Acosta, A.B. Blanco, J.A. Gallego-Juárez: Food drying process by power ultrasound. Ultrasonics 44 (2006) e523-e527.

13. J.A. Gallego, G. Rodriguez, J.C. Gálvez, T.S. Yang: A new high-intensity ultrasonic technology for food dehydration. Drying Technology 17 (1999) 597-608.

14. Y. Zheng, Q.K. Zhang: Simultaneous measurement of gas and solid holdups in multiphase systems using ultrasonic technique. Chemical Engineering Science 59 (2004) 3505-3514.

15. P. Collas, M. Barmatz, C. Shipley: Acoustic levitation in the presence of gravity. Journal of the Acoustical Society of America 86 (1989) 777-787.

16. D.P. Gautam, L.F. Donald: Acoustically aided separation of oil droplets from aqueous emulsions. Chemical Engineering Science 59 (2004) 3183-3193.

17. J.A. Gallego-Juárez, G. Rodriguez-Corral, L. Gaete-Garreton: An ultrasonic transducer for high power applications in gases. Ultrasonics 16 (1978) 267-271.

18. I. Gonzalez, J. Rodríguez, I. Garmendia, J.A. Gallego-Juárez: Application of high intensity air-borne ultrasound for debubbling liquid coating layers. Ultrasonics 44 (2006) e529-e532.

19. E. Riera, J.A. Gallego, T.J. Mason: Airborne ultrasound for the precipitation of smokes and powders and the destruction of foams. Ultrasonics Sonochemistry 13 (2006) 107-116.

20. G. Rodriguez, E. Riera, J.A. Gallego, V.M. Acosta, A. Pinto, I. Martinez, A. Blanco: Experimental study of defoaming by air-borne power ultrasonic technology. Physics Procedia 3 (2010) 135-139.

21. H. Yamamori, T. Ito, K. Ohya, H. Banno: Characteristics of bolt-Clamped Langevin type ultrasonic transducer with square radiating surface. Journal of the Physical Society of Japan 29 (1990) 167-169.

22. M.D. Waller: Vibrations of free square plates: part I. Normal vibrating modes. Proceedings of the Physical Society 51 (1939) 831-844.

23. M.D. Waller: Vibrations of free square plates: part II. Compounded normal modes. Proceedings of the Physical Society 52 (1940) 452-455.

24. X.P. He, X.L. Yan, N. Li: Directivity pattern of the sound radiated from axisymmetric stepped plates. Journal of the Acoustical Society of America 140 (2016) 1387-1396. 